\title{
Civilisations
}

Revue internationale d'anthropologie et de sciences

humaines

$41 \mid 1993$

Mélanges Pierre Salmon II

\section{Contribution à l'histoire jusque 1934 de la création de l'institut des parcs nationaux du Congo belge}

Jean-Paul Harroy

\section{(2) OpenEdition \\ Journals}

Édition électronique

URL : http://journals.openedition.org/civilisations/1732

DOI : 10.4000/civilisations. 1732

ISSN : 2032-0442

Éditeur

Institut de sociologie de l'Université Libre de Bruxelles

Édition imprimée

Date de publication : 1 septembre 1993

Pagination : 427-442

ISBN : 2-87263-094-5

ISSN : 0009-8140

Référence électronique

Jean-Paul Harroy, « Contribution à l'histoire jusque 1934 de la création de l'institut des parcs

nationaux du Congo belge », Civilisations [En ligne], 41 | 1993, mis en ligne le 30 juillet 2009, consulté le

30 avril 2019. URL : http://journals.openedition.org/civilisations/1732 ; DOI : 10.4000/

civilisations. 1732

Ce document a été généré automatiquement le 30 avril 2019.

(c) Tous droits réservés 


\title{
Contribution à l'histoire jusque 1934 de la création de l'institut des parcs nationaux du Congo belge
}

\author{
Jean-Paul Harroy
}

\section{Preambule}

1 Le présent article m'a été souvent demandé par des personnes inquiètes de ce qu'aucune synthèse importante ait jamais décrit les étapes, amorçées vers 1920, qui ont conduit le 26 novembre 1934 à la création de l'Institut des Parcs nationaux du Congo Belge (I.P.N.C.B.). Et je me juge un peu responsable de cette lacune, étant devenu aujourd'hui probablement le seul survivant des derniers témoins de la fin de cette époque. J'exerçai, en effet, à partir du 25 mars 1935 la direction de cette institution jusqu'au début 1948, rencontrant et interrogeant de nombreux acteurs de la décennie précédente : Prof. Van Straelen, Colonel Hackars, Colonel Hoier, etc, et ayant à ce moment accès à tous les dossiers anciens. N.B. Je n'ai pas jusqu'ici réussi à retrouver à Bruxelles les archives de l'I.P.N.C.B. Il reste donc encore matière à de nouvelles recherches et à de nouvelles rédactions.

2 Le titre de cet article dédié à mon ami Pierre Salmon, l'un des meilleurs historiens de l'Afrique $^{1}$, veut clairement indiquer les limites que j'ai tenu à assigner à mon texte : une contribution à l'Histoire. D'autres publications devraient la compléter, dont peut-être en premier lieu le bilan détaillé et commenté des remarquables réalisation de l'I.P.N.C.B. entre 1934 et 1960.

Me cantonnant à une relation historique de la création de cet Institut, je renoncerai donc à toute description biogéographique des réserves naturelles sous revue et à propos desquelles le lecteur peut d'ailleurs consulter une très abondante bibliographie, quand ce ne serait que les quelque 350 publications de l'Institut lui-même.

4 C'est au début de la seconde moitié du dix-neuvième siècle que prit corps la préoccupation: dans maintes régions du globe, une chasse excessive menace de disparition d'importantes collectivités de faune sauvage - on s'est inquiété plus tard des 
forêts, des paysages, des éco-systèmes - et il s'avère nécessaire de prendre des mesures de protection en conséquence. A l'échelle mondiale, les premières réalisations importantes se localisèrent en 1872 aux USA avec, pour premier jalon majeur, le Yellowstone National Park.

Les mêmes phénomènes : massacres, inquiétudes, réactions du monde scientifique et des autorités publiques se manifestèrent un peu plus tard en Afrique Equatoriale. Un nom s'y attache dès 1888, celui du Major allemand von Wissmann, qui, centrant d'abord ses efforts sur les éléphants, parvint à amener le Roi Léopold II à créer, dès cette époque lointaine, des réserves à éléphants dans l'Etat Indépendant du Congo, notamment au Kasaï.

Des voix s'élevèrent bientôt pour réclamer davantage : la création de réserves générales de chasse, où toute la faune serait protégée. Le principe en fut admis internationalement en 1900, à la Conférence Internationale de Londres pour la protection des animaux sauvages africains. C'est en vertu de cette Conférence que furent créés par la suite au Congo Belge quelques bonnes réserves générales de chasse dont les plus notoires, Kivu, Rwanda, Uele, Katanga, servirent beaucoup plus tard de soubassement aux quatre premiers parcs nationaux Albert, Kagera, Garamba, Upemba.

7 En 1909, peu avant la mort de Léopold II, le Prince Albert rendit visite au jeune Congo Belge et, rentré en Belgique, il joignit sa voix à celle des partisans de la création dans la Colonie belge de réserves intégrales de chasse.

8 Au début du siècle était également apparu le problème du Gorille des Montagnes au Kivu. Le premier spécimen de ce célèbre primate fut abattu en 1902 sur les rampes du volcan éteint Sabyinio, dans le Nord du Rwanda, par le Capitaine allemand von Beringe. Décrit en 1903 à Berlin par le zoologiste Matschie, il fut dénommé Gorilla beringei Matschie.

Cette annonce fit sensation, surtout qu'elle ouvrait la voie à la controverse : Gorille des Plaines, déjà connu, et Gorille des Montagnes, espèce nouvelle ? Et bientôt parvinrent à Bruxelles et à Léopoldville de nombreuses demandes d'autorisations d'abattage de ce précieux mammifère. Un Belge, M. Brichart, est connu pour avoir en 1906 tué un gorille "sur les versants Sud du Ruwenzori" (?). En 1912, un chasseur britannique renommé, M. Burbridge, fut à son tour autorisé à en récolter quatre, et l'année suivante la même faveur fut accordée à la Mission du Duc de Sudamanie, Prince de Suède. Cette dernière expédition fit malheureusement très mauvais usage des quatorze permis qui lui avaient été octroyés, et valut de fortes critiques internationales aux autorités belges, provoquant une beaucoup plus grande sévérité dans l'octroi de ces autorisations de récolte.

Vers 1916-18, en pleine guerre mondiale, les Américains à leur tour demandèrent des autorisations, au profit du Musée National d'Histoire naturelle de New-York, représenté par son déjà célèbre taxidermiste Carl Akeley. Ce dernier poursuivait essentiellement un objectif : rassembler le matériel nécessaire à la confection dans son Musée d'un de ses célèbres dioramas, cette fois dédié au Gorille des Montagnes. Accessoirement, il songeait aussi à contribuer à la préparation de la création au Kivu d'un "Gorilla Sanctuary".. "to make the World safe for Gorillas", expression vite devenue classique.

11 Akeley se rendit au Kivu en 1920. Simultanément était autorisé à récolter et observer à ses côtés un zoologiste anglais Alexander Burns du British Museum of Natural History. A son retour aux USA en 1921, Akeley publia un livre "In Brighest Africa", fournissant une première estimation du nombre des Gorilles de Volcans (il parlait de 200. Aujourd'hui on 
croit : 250) et enrichissant notoirement les connaissances sur la biologie et les moeurs des primates étudiés par lui.

L'influence de son "best-seller" se mélangea alors aux retombées de la visite à sensation que venait de rendre au Yellowstone N.P., en 1919, le Roi Albert, à ce moment tout auréolé de sa gloire de Roi Chevalier ayant tenu tête à l'agression allemande de 1914. Au cours d'un feu de camp mémorable (un demi-siècle après le célèbre camp-fire de Washburn à Madison), et au même endroit, le Roi, fortement soutenu par d'éminents "écologistes avant la lettre", dont John C. Merriam (Carnegie Institution) et Fairfield Osborn (American Museum of Natural History) et par l'Ambassadeur de Belgique à Washington, Baron E. Cartier de Marchienne, prononça sa formelle intention de voir reproduit au Congo belge le modèle américain du "Parc National".

Les années qui suivirent virent se créer aux Etats-Unis un "Comité Américain" de ce que l'on commençait déjà à appeler le "Parc National Albert" (animateurs, on s'en doute : J.C. Merriam et F. Osborn). Simultanément, pour enrichir le dossier, une nouvelle mission Akeley fut programmée, financée (intervention Eastman) et organisée pour 1926. Et en Belgique, pour déférer au voeu royal que le Palais ne perdait pas de vue, le Ministère des Colonies (Directeur Général E. Leplae,) stimulé et conseillé par divers naturalistes, dont, déjà, le Prof. V. Van Straelen, Directeur du Musée Royal d'Histoire Naturelle de Belgique, travaillait, non sans se heurter à des difficultés d'ordre administratif ou juridique - voire quelques hostilités à courte vue - à la préparation du Décret du 21 avril 1925 par lequel le Roi des Belges allait créer au Kivu le "Parc National Albert" N.B. C'était le premier territoire protégé d'Afrique à porter le nom de Parc National, les Sud-Africains, pourtant très avancés en la matière, n'ayant donné qu'en 1926 le nom de "Krüger national Park" à leur excellente Sabie Game Reserve au Transvaal.

Conformément à la législation, le projet de décret fut soumis au Conseil Colonial de Bruxelle, 28 mars 1925. Ce Collège avait réservé à la proposition un accueil enthousiaste et certains membres avait été jusqu'à demander que la superficie du premier "Parc National Albert" dépasse les 200.000 hectares, englobant, outre les 10.000 hectares du "Gorilla Sanctuary" Mikeno-Karisimbi-Bishoke, divers territoires voisins, dont ce qui allait devenir la réserve de Chasse de Rutshuru (14 août 1925) et la réserve de la Ruindi, vaste plaine giboyeuse atteignant la rive Sud du Lac Edouard.

Un fascicule publié en 1934 précisait cette intention de 1925 : "ajouter encore une série de concessions, de missions, de cultures indigènes, de villages", qu'allaient d'ailleurs, aussi classer peu après la réserve du 14 août 1925.

Ces propositions trop ambitieuses du Conseil Colonial effrayèrent le Ministre des Colonies. Les justifications de sa prudence, on les lit dans un texte de Paul Brien (Biographie Nationale) $^{2}$ : "l'insuffisance de nos connaissances de la région, de ses limites réelles, de sa topographie, de sa démographie, de ses ressources naturelles n'y permettait pas une administration réelle et efficace". On s'en tint donc pour le décret du 21 avril 1925 aux 10.000 hectares du "Gorilla Sanctuary". Et l'on décida qu'une mission sur les lieux s'imposait pour 1926.

Une brève parenthèse se justifie ici pour évoquer l'essor que prenait pendant la décennie 1920-1930, un courant, surtout européen, de volonté de protection internationale de la 
Nature. En 1910, le Prof. suisse Paul Sarrasin avait déjà réussi, avec le soutien de l'Union internationale des Sciences biologiques (UISB), à jeter les bases d'une collaboration transfrontière en cette matière. Mais la guerre et le Conseil Fédéral helvétique firent perdre quinze années à la gestation du projet. Et ce dernier ne refit surface qu'en 1925 avec comme promoteurs autour du tenace Paul Sarrasin, le Hollandais Peter Gerbrand van Tienhoven, le Polonais Michel Sielecki, quelques autres naturalistes et trois Belges, le Prof. Jean Massart, le Prof. Victor Van Straelen et, on dirait presque surtout, un jeune brillant zoologiste (Dr. Sc. ULB 1922), Jean-Marie Derscheid.

Ce dernier y consacra tant d'efforts que, lorsqu'en juillet 1928 fut créé à Bruxelles, avec pour siège la Fondation Universtaire rue d'Egmont, un "Office International de Documentation et de Corrélation pour la Protection de la Nature" (OIPN), c'est lui qui en devint le Secrétaire Général, avec pour Président P.G. van Tienhoven.

Toujours pendant la même période 1923-1927, le Dr. Derscheid, alors basé à Tervueren comme adjoint au Dr. Schouteden, Directeur de la Section des Sciences Naturelles du Musée, partagea son temps entre de premiers enseignements, ses tâches OIPN (en 1926, on créa un Comité Belge de la protection internationale de la Nature dont il fut également Secrétaire Général) et une dynamique participation à la promotion du projet en gestation "Parc National Albert".

On aura remarqué que la préparation et la promulgation à Bruxelles du Décret du 21 avril 1925 coïncidaient chronologiquement avec la période où le Musée d'Histoire Naturelle de New-York préparait sa nouvelle mission Akeley au Kivu.

21 Très logiquement, après des contacts où intervinrent l'Ambassadeur Baron Cartier de Marchienne et plusieurs membres du Comité Américain du P.N.A., il fut convenu que la Mission Akeley serait accompagnée par "un scientifique belge chargé de ramener un maximum d'informations pouvant conduire à la mise en application sur le terrain des prescriptions du décret du 21 avril 1925". Le choix se porta, on s'en doute, sur J.-M. Derscheid. On insista sur le caractère prioritairement scientifique de la mission dont il était revêtu.

22 Le chargé de mission Derscheid s'embarque à Marseille le 2 septembre 1926. Le 24 du même mois, il est accueilli à la gare de Nairobi par Madame Akeley dont le mari se relève difficilement d'une fièvre aigüe contractée deux mois plus tôt au Tanganyika Territory. L'entente s'établit très vite entre les membres de la mission (six en tout, y compris Derscheid). Les derniers préparatifs durent jusqu'au 16 octobre. Pendant ces quelques semaines, J.-M. Derscheid est à Nairobi, officiellement invité au "Bureau de Chasse gouvernemental" où il est documenté sur "l'organisation de la surveillance, les mesures prises, les règlements sur la conservation du gibier, les lois relatives à la Chasse depuis 1898 ainsi que leur historique" (Journal du Chargé de Mission).

Le 6 novembre, le groupe est à Rutshuru. Akeley est toujours souffrant. Avec un courage magnifique, il refuse de renoncer et même de s'arrêter quelques jours pour se reposer. L'exploration proprement dite des Volcans commence le 8 novembre, au départ de ce qui fut longtemps un poste détaché du P.N.A. (et son potager): Nyakibumba. La mission campe le 15 à Kabara, dans la selle entre Mikeno et Karisimbi. L'état d'Akeley empire encore. Il meurt dans l'après-midi du 17 novembre 1926. J.-M. Derscheid assume toutes les responsabilités découlant de ce catastrophique décès, règles les funérailles sur place, soigne l'établissement de la tombe ${ }^{3}$. Il organise le retour immédiat en Amérique de Mme Akeley et des autres membres de la Mission. En ce qui le 
concerne, il décide logiquement de continuer seul la tâche de reconnaissance que lui a confiée le gouvernement belge.

Les conditions climatiques sont exceptionnellement rudes, ses travaux - notamment de cartographie - s'effectuant aux prix d'efforts épuisants. Fin décembre, il doit fuir le brouillard et les averses des volcans et en profite pour consacrer janvier 1927 à explorer la partie septentrionale de la Réserve de Chasse (24/2/25), les "Plaines du Lac Edouard" dont il sait que le Palais prévoit l'inclusion ultérieure dans un P.N.A. agrandi.

Pendant ce premier trimestre 1927 passé sur le terrain, Derscheid a eu probablement aussi des contacts préliminaires avec les autorités locales du Service Territorial et de l'Agriculture pour fixer quelques principes de départ, notamment quant aux problèmes de délimitation voire abornement des zones protégées, de mise au travail de premiers gardes P.N.A., du tracé de certains sentiers ou pistes automobiles pour la Réserve de Chasse du 24-2 (Ruindi) et du 14-8-25 (Rutshuru). Le décret de 1925 prévoit, en effet, que l'administration du P.N.A. est confiée au Gouverneur Général. Ce dernier, pour exécuter et coordonner ces nouvelles tâches, envisageait déjà à ce moment la création d'une nouvelle fonction, celle de Conservateur du P.N.A. Ce poste fut créé dès l'année suivante. Son titulaire était choisi homme d'action beaucoup plus qu'expert en organisation et gestion. Il se nommait Clément Hemeleers. Son intrépidité était légendaire. Un article du 23 octobre 1931 de l'hebdomadaire bruxellois "Pourquoi Pas ?" le décrit: "singulier et délicieux garçon qui s'était donné pour métier de vivre au milieu des lions. Il faisait cela très bien. Le jour, il les photographiait. La nuit, il dormait au milieu d'eux, sans fusil, avec pour tout épouvantail son (sic) moustiquaire et ses ronflements... On lui avait dit de surveiller les bêtes. Il surveillait surtout les hommes qui faisaient mine de les tuer...".

La mission Derscheid au Kivu prend alors fin en juillet 1927. Il rentre à Bruxelles et dépose son rapport le 8 octobre suivant.

Outre la moisson d'observations attendues, ce rapport contient une proposition formelle : la création d'une institution autonome, distincte du Gouvernement Général, à qui confier la gestion du P.N.A. Cette suggestion hardiement innovatrice a-t-elle été imaginée à ce moment par J.-M. Derscheid? Ou était-elle déjà "dans l'air" depuis quelque temps, en Belgique, voire aux USA. ? J'opterais pour la seconde hypothèse.

Toujours est-il que l'année 1928 n'allait donc pas tant être marquée par d'importantes réalisations au Kivu - sauf l'apparition d'Hemeleers - que par la préparation d'un nouveau décret (qui va être signé le 9 juillet 1929) et dont les deux caractéristiques majeures allaient être l'établissement de l'institution autonome P.N.A. et la considérable extension de la superficie du P.N.A. jusqu'au Lac Edouard.

30 Le projet fut soumis au Conseil Colonial le 28 juin 1929. Celui-ci accepta sans réserve le principe novateur de structure d'autonomie de même qu'une considérable extension territoriale (nouvelle superficie 292.098 hectares) déterminée par un document cartographique annexé au Décret et intitulé "Parc National Albert; Kivu-Ruanda; Délimitation 1929. Décret du 9 juillet 1929".

31 Ce schéma fait apparaître une nouvelle distinction définie dans l'article 2 du décret "La partie des territoires du Congo belge et du Ruanda-Urundi réservée à la poursuite à des fins scientifiques de l'Institution comprend des réserves naturelles intégrales et des territoires annexes". Les articles 6 et 7 suivants précisent les régimes, sévère pour les réserves intégrales, beaucoup plus laxiste pour les territoires annexes, applicables dans les territoires protégés. 
La carte fait état de trois groupes de réserves intégrales: $1^{\circ}$ le Secteur Septentrional (plaine au Sud du Lac Edouard) 84.272 ha, $2^{\circ}$ les Secteurs Centraux (Mikeno etc., partie au Kivu, partie au Ruanda) 51.480 ha et Oriental (volcans éteints au Ruanda) 8.408 ha et enfin $3^{\circ}$ le Secteur Occidental (volcans actifs du Kivu : Nyamuragira et Nyiragongo) 57.552 ha. soit au total : 201.712 ha de réserve naturelle intégrale.

A quoi s'ajoutent 90.386 ha de territoires annexes, dont 55.902 ha au Binza (plaine de la Rutshuru, au Sud du Secteur Septentrional), 18.644 ha au Sud des Secteurs Central et Occidental ( 7.820 au Kivu et 10.874 au Ruanda) et enfin, en bordure S.-E. des Secteurs Central et Oriental, une dernière portion de 15.840 ha. Total Général : 292.098 ha.

Le caractère "parastatal" du nouveau P.N.A. impliquait une grande audace politique et constitutionnelle puisque des étendues importantes de territoires coloniaux ou sous mandat S.D.N. allaient cesser de relever de l'autorité exclusive du Gouverneur Général, des gouverneurs et des Territoriaux locaux, pour être désormais "administrés" par une "Commission Administrative" d'une institution autonome bénéficiant de la personnalité civile. Plus hétérodoxe encore, cette Commission aux pleins pouvoirs allait en son sein compter, sur un total de 18 membres, six personnalités étrangères concernées par la protection de la Nature. Cette disposition visait à mettre en exergue le caractère international et prioritairement scientifique (cf. Discours Royal d'Installation de la Commission le 19 octobre 1931) que la Belgique et son Souverain entendaient conférer à la nouvelle institution.

Dépendait alors de la Commission, pour assurer la gestion courante, un Comité de Direction présidé par le Président de la Commission, composé d'une demi-douzaine de Membres Belges où, très logiquement, figurait le nom de Victor Van Straelen.

Enfin un directeur complétait, avec voix consultative, ces deux collèges supérieurs. La nomination de J.-M. Derscheid comme directeur ne fit à l'époque l'objet d'aucune objection. Quant à la Présidence, elle fut attribuée au Prince de Ligne, dans un contexte un peu trouble que caractérise l'article précité du 23 octobre 1931 du "Pourquoi Pas ?"... pour matelasser les choses (sic) on mit à la tête de ces docteurs un homme qui n'était pas du métier : on leur donna le Prince de Ligne parce qu'il allait au Kivu chaque année et qu'au moins lui ne jalouserait la place de personne".

37 En son article 5, le Décret constitutif de 1929 prévoyait "il peut être créé un corps spécial de conservateurs et de gardes". Cette disposition concrétisait ce qui, comme dit plus haut, allait pendant des années entretenir des tensions, voire des sources de conflits, entre les représentants sur place, portant titre de conservateur, de la nouvelle institution P.N.A. et les autorités locales, principalement Clément Hemeleers, qui antérieurement avaient seuls assuré l'administration du Parc.

Fut ainsi engagé par le P.N.A. le $1^{\text {er }}$ novembre 1929 (donc sans attendre) le colonel danois Rasmus Hoier, ancien de la Force Publique et des Campagnes de l'Est-Africain.

$\mathrm{Du} 1^{\mathrm{er}}$ janvier au $1^{\mathrm{er}}$ mai 1930, le Président et le Directeur entreprirent ce que le Ministère qualifia de "seconde mission". Certains aspects de celle-ci ne furent pas du goût de tout le monde, ouvrant la voie à des remarques du genre "Pourquoi Pas ?" quant à l'inexpérience du Président et justifiant des allusions en demi-teinte de Paul Brien (sa Biographie, p. 225) : "tout était à créer, il fallait apaiser des réticences, vaincre des oppositions. Le naturaliste Derscheid y était peu préparé. Son initiation aux opérations administratives était récente encore. Néanmoins, il fallait aller de l'avant malgré les obstacles, l'inexpérience, les tâtonnements, les hésitations, les erreurs inhérentes à toute oeuvre 
nouvelle. Derscheid eût dû être guidé et soutenu. "Et pointaient ainsi des orages qui allaient fin 1933 conduire à l'effacement du pionnier Derscheid.

Je n'étonnerai personne ni ne divulguerai de grands secrets en révélant que les mécontentements croissants éprouvés à l'égard de cette évolution peu satisfaisante des premières réalisationns in situ du P.N.A., furent surtout ressentis par le Roi-fondateur et son conseiller scientifique d'alors le Prof. Victor Van Straelen. Sous leur double impulsion furent préparées au Ministère plusieurs décisions importantes dont certaines se concrétisèrent dès le 21 novembre 1931. A cette époque, coïncidant avec de premiers renouvellements triennaux de Membres de la Commission et du Comité de Direction, la Présidence de l'Institution fut assumée par S.A.R. le duc de Brabant, ayant à ses côtés un Vice-Président, en l'occurrence Victor Van Straelen. Etaient à cette date Membres du Comité de Direction le Prof. R. Bouillenne (Lg), Paul Charles, Administrateur Général des Colonies, le Prof. Robyns (Lv), le Prof. Schoep (Gd), H. Schouteden (Tervueren) et J. Willems, Directeur du F.N.R.S. ; le Dr. J.-M. Derscheid restait directeur.

Début 1932, le Roi Albert décida de se rendre sur place, en compagnie de Victor Van Straelen (23 mars au 25 avril). C'était, on s'en souvient, l'année de l'expédition F.N.R.S. au Ruwenzori. Au cours de ce périple, où le Roi entreprit l'ascension du Mikeno, furent prises d'autres résolutions importantes, notamment pour lancer l'exploration scientifique du P.N.A. et l'organisation de l'importante mission de récoltes et d'observations de l'herpétologue Gaston-F. de Witte.

Fin 1932, après son inspection sur place, le Roi Albert avait également demandé au Gouverneur Général, à ce moment le Général Tilkens, de lui désigner une personnalité de grand format qui, avec le titre de Conservateur P.N.A., allait pouvoir se charger de certaines tâches importantes et délicates. Le choix se porta sur le Colonel Henri M. Hackars, ancien Commissaire de District d'Irumu. Celui-ci, complétant et même coiffant le Colonel Hoier, exerça par intermittence les fonctions de Conservateur à Rutshuru, régla de nombreux problèmes avec les autorités locales, procéda à des enquêtes de rachats de droits indigènes et accomplit une série de reconnaissances préliminaires à l'éventuelle ultérieure extension du P.N.A. vers le Nord (plaine de la Semliki, massif du Ruwenzori). Il se livra aussi, cette fois loin du P.N.A., à de premières négociations préalables à de futures créations en Uele (Garamba) et au Katanga (Upemba). Son habileté, avec en filigrane l'appui du Roi et du Gouverneur Général, fit merveille.

43 L'année 1933 vit alors se dessiner l'amorce d'un professionalisme de gestion qui allait utilement remplacer l'aimable amateurisme de 1929-1932. Le Duc de Brabant, qui présidait en personne toutes les séances du Comité et de la Commission, joua un rôle important dans cet encourageant virage annonciateur de l'ère de grande rigueur qu'allait bientôt imposer la poigne d'un Van Straelen. En 1933, il se rendit sur place, accompagné de la Princesse Astrid. Le 16 novembre 1933, à l'African Society de Londres, le Prince prononça un mémorable discours à l'occasion de la conférence internationale pour la protection de la faune et de la flore en Afrique.

Enfin, c'est pendant les dernières semaines de 1933 que se produisit le gros incident Derscheid, à propos duquel je resterai délibérément discret, et qui, dans un contexte pénible, mit tristement fin à la carrière P.N.A. du pionnier des années vingt ${ }^{4}$. Albert, qui contraignit le nouveau Roi Léopold III à renoncer à sa présidence P.N.A., 
fonction à laquelle fut aussitôt nommé le Prof. Van Straelen. Deuxième événement méritant mention: la désignation début 1934 d'un nouveau directeur, excellent organisateur, qui avait déjà marqué l'institution de son empreinte bénéfique lorsqu'en fin d'année un autre incident requérant discrétion mit fin à son engagement. Enfin, c'est le 26 novembre 1934 que fut créé par décret "L'Institut des Parcs Nationaux du Congo Belge" (I.P.N.C.B.), se substituant à l'institution P.N.A., notamment pour permettre la création d'autres parcs nationaux en Afrique belge. Les innovations de 1929 n'étaient pas remises en question et la surface du P.N.A. subissait quelques nouvelles extensions. De plus, un nouveau Parc National était créé, cette fois au Ruanda: le P.N.K. Parc National de la Kagera (devenu de l'Akagera après 1962), 250.000 ha. dont 80.000 de territoire-annexe.

Pour 1935, je me permets de considérer comme un fait notoire ma nomination le 25 mars à la Direction de l'Institut. Echaudés par deux mésaventures récentes, les responsables de cette nomination me refusèrent le titre de Directeur, remplaçant ce dernier par "Secrétaire du Comité de Direction". A mon arrivée rue Montoyer, j'y trouvai exerçant l'intérim le Colonel Hackars, déjà cité et louangé ci-dessus. Mes premières armes se firent à ses côtés. Je retirai un bénéfice immense de cette collaboration amicale d'une année avec un homme exceptionnel de rigueur morale et d'expérience africaine. Et puisque j'en suis au "moi haïssable", que le lecteur m'autorise à procurer ici quelques détails personnels : guerre de 1940 passée en Belgique, intérim de formation sur le terrain : un an au P.N.A. (1937-38) et une autre année au P.N. de la Garamba (Uele) en 1947-48. Nommé à ce moment (1948) Secrétaire Général de l'I.R.S.A.C. (Institut pour la Recherche scientifique en Afrique Centrale), je fus remplacé à la Direction de l'I.P.N.C.B. par l'entomologiste Henri De Saeger, lequel resta en fonction jusqu'à l'indépendance du Congo en 1960 et du Ruanda, en 1962. Autre événement de 1935 : la signature par le Roi Léopold III du décret du 12 novembre ajoutant au P.N.A. ses secteurs Semliki et Ruwenzori et portant sa surface à quelque 810.000 ha.

Respectueux du cadre limité du présent article, je laisserai maintenant à d'autres écrits, dont beaucoup existent déjà, l'analyse de la remarquable croissance que connut l'I.P.N.C.B. de 1934 à 1960 sous la conduite vigoureuse et éclairée de Victor Van Straelen. On y verra la création le 17 mars 1938 du Parc National de la Garamba (Uele, 500.000 ha) et celle, le 15 mai 1939, du Parc National de l'Upemba (Katanga, 1.730.000 ha). On attend surtout une autre nécessaire synthèse analysant les principes fondamentalement respectueux de l'écologie sur lesquels furent maintenus pendant ce quart de siècle l'aménagement scientifique des réserves, leur prudente ouverture au tourisme, leur exploration scientifique : trois grandes missions, Mission de Witte, 1933-35, au P.N.A., Mission De Saeger au P.N.G. 1942-52, et Mission Verheyen-de Witte à l'Upemba, 1946-49, 25 missions de moindre ampleur, 87 chercheurs isolés belges ou étrangers.

50 La préparation des récoltes, leur envoi aux spécialistes, l'adaptation des manuscrits et leur impression étaient dirigés par G.-F. de Witte, chef de la Section Scientifique de l'Institut. A l'heure des bilans, après l'indépendance du Zaïre, le total des publications de l'I.P.N.C.B. approchait des 350 livres ou fascicules.

51 Enfin, j'omettrai encore délibérément l'évocation des faits postérieurs à 1960, générateurs du relativement très bon état de santé - malgré les incertitudes politiques des actuels parcs nationaux zaïrois. Il s'agit d'une autre épopée, toujours en cours, au fil de laquelle quatre nouveaux parcs nationaux zaïrois furent créés autour des années 1970. S'y rattachent essentiellement les noms du Président Mobutu, très concerné par la conservation de l'Environnement, du zoologiste belge Jacques Verschuren qui, fait à 
souligner, dirigea de 1969 à 1974 l'Institut zaïrois pour la Conservation de la Nature (I.Z.C.N.) et de l'actuel Président-Délégué Général de l'I.Z.C.N., l'excellent écologiste et organisateur zaïrois Mankoto wa Mbaelele.

\section{ANNEXES}

\section{Les Parcs Nationaux du Congo Belge en 1960}

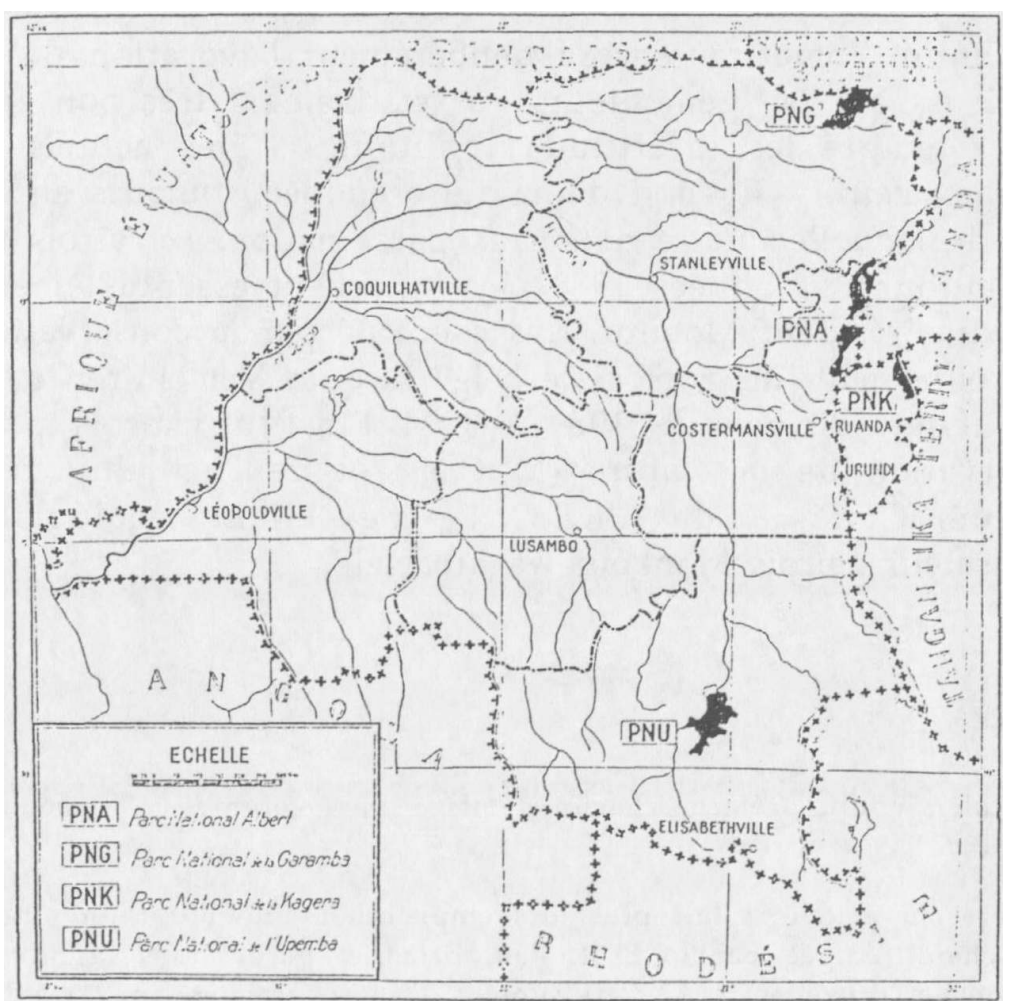

\section{NOTES}

1. Il a bien voulu préfacer chacun de mes épais volumes : "Rwanda" puis "Burundi".

2. Le présent article a fait plusieurs emprunts à une biographie de J.-M. Derscheid rédigée par le Prof. Paul Brien et parue dans la Biographie Nationale (Bruxelles), t. XXXVII, suppl. t. IX, fasc. premier, pp. 211 à 235.

3. Tombe que j'ai restaurée et fleurie en avril 1937 et qui est toujours très bien entretenue par les Zaïrois.

4. La famille Derscheid détient des archives de Jean-Marie auxquelles, on devine pourquoi, je n'ai jamais eu accès, mais que d'aucuns ont pu consulter, dont l'historien rundi Joseph Gahama. Héros 
de la Résistance, J.-M. Derscheid fut décapité à la hache le 13 mars 1944 dans la prison de Brandebourg, sur ordre de Himmler.

\section{RÉSUMÉS}

Director of the National Parks Institute of the Belgian Congo from 1935 to 1948, the author describes the steps undertaken at the beginning of the $20^{\text {th }}$ century which led to the creation of the Institute in 1934. With the discovery of the Kivu mountain gorillas in 1902, hunters were the first to become interested in the Congo wildlife. But following the visit of the taxidermist Carl Akelei to Kivu in 1920 for the benefit of the National History Museum of New York and the appearance of his book "In Brightest Africa" in 1921, coupled with the visit of King Albert to Yellowstone National Park in the United States in 1919, the idea of creating a reproduction in the Congo of the American "National Park" took shape in Belgium and in 1925, the "Albert National Park" was created at Kivu. This was the first protected territory in Africa to be called a National Park. Following the increase in number and extent of the National Parks in the Congo, the National Parks Institute of the Belgian Congo was set up in 1934.

\section{AUTEUR}

\section{JEAN-PAUL HARROY}

Faculté des Sciences sociales, politiques et économiques - Université Libre de Bruxelles Belgique 